\title{
Single scattering particles based analytical techniques
}

\author{
LIU Yue ${ }^{1} \&$ HUANG ChengZhi ${ }^{1,2 *}$ \\ ${ }^{1}$ Education Ministry Key Laboratory on Luminescence and Real-Time Analysis, School of Chemistry and Chemical Engineering, \\ Southwest University, Chongqing 400716, China; \\ ${ }^{2}$ College of Pharmaceutical Science, Southwest University, Chongqing 400716, China
}

Received October 21, 2012; accepted December 14, 2012; published online February 28, 2013

\begin{abstract}
Single scattering particles, especially noble metal (plasmonic) nanoparticles, based analytical techniques are attractive recently and becoming the research focus of the light scattering analytical techniques. In this mini review, we summarize the single scattering particles based analytical techniques in the past decade including single scattering particles counting, single plasmonic nanoparticles sensing, and single plasmonic nanoparticles tracking/imaging. We emphasize the discussion on the single plasmonic nanoparticles sensing that combines with dark-field microscopy and resonant Rayleigh scattering spectroscopy.
\end{abstract}

single particles, light scattering, analytical techniques, plasmonic nanoparticles, dark-field

Citation: $\quad$ Liu Y, Huang C Z. Single scattering particles based analytical techniques. Chin Sci Bull, 2013, 58: 1969-1979, doi: 10.1007/s11434-013-5707-4

Scattering particles exist everywhere from the nature to the nanoworld, such as dusts, fogdrops, chromophore aggregates, and plasmonic nanoparticles [1,2]. Using some of these scattering particles, resonance light scattering analytical technique has been developed for biochemical analysis. For example, chromophore aggregates were firstly used for biomolecules detection, such as nucleic acids [3,4]. This technique was always carried out by simultaneously scanning the excitation and emission monochromator a spectrofluorometer. Thus, the light scattering spectrum of chromophore aggregates can be recorded and used for analytical purposes. Thereafter, Mirkin and co-workers [5-7] used plasmonic nanoparticles with excellent scattering property for biochemical assays through scattering light color differentiation between disperse nanoparticles and targets induced aggregated nanoparticles. However, above mentioned analytical procedures always focused on an ensemble of scattering particles and suffered from the disadvantages of high sample-consuming, low sensitivity, low spectral resolution, and rare application in biosystem. These drawbacks prevent practical use of plasmonic nanoparticles in most cases.

Accompanying with the development of new optical de-

*Corresponding author (email: chengzhi@swu.edu.cn) tectors, particularly imaging detection system, scattering signals from single particles can be easily detected. Thus, single scattering particles based analytical techniques have been developed for biochemical analysis and are becoming the mainstream of light scattering analysis recent years. These techniques minimize the sensing platforms to a single scattering particle instead of an ensemble of scattering particles and seem to provide the possibility to address the above mentioned drawbacks. In this mini review, we summarize the single scattering particles based analytical techniques in the past decade, including single scattering particles counting, single plasmonic nanoparticles sensing, and single plasmonic nanoparticles tracking/imaging. Plasmonic nanoparticles intrinsically possess excellent scattering property and have been used widely used as single particles sensors. Our discussion is emphasized on single plasmonic nanoparticles analysis based on its dielectric environment change, plasmon coupling, plasmon resonance energy transfer, and chemical reaction using dark-field microscopy and resonant Rayleigh scattering spectroscopy.

\section{Single scattering particles counting}

Single scattering particles counting, as its name suggests, is 
an analytical technique by counting the number of single scattering particles using the equipment that can detect the light scattering signals. In 2003, we developed a light scattering imaging technique as a pioneering work for the detection of proteins by counting single scattering particles of $\alpha, \beta, \gamma, \delta$-tetrakis ( $p$-sulfophenyl)porphyrin (TPPS4) aggregates [8]. In the absence of proteins, free TPPS4 shows low scattering light (Figure 1(A)), and it is hard to observe the light scattering particles. While in the presence of proteins, which also has low scattering light (Figure 1(B)), J-aggregation of TPPS4 occurs, resulting in strongly enhanced light scattering signals (Figure 1(C) and (D)).

Herein, a home-built optical system was introduced to counting the scattering particles, which were hold in a small cell and simply illuminated by a laser beam and a fluorescence microscope coupled with a cooled charge-coupled device (CCD) were employed to observe the light scattering of the aggregates. It is very easy to observe the J-aggregation TPPS4 particles in the focus plane and the light scattering images then could be easily captured with a CCD camera. The aggregates observed in the focus plane could be expressed as white spots (Figure 1). As the concentration of protein gets higher, the white spots increased, and the counts of the spots were linear to the concentration of proteins, which forms the basis of proteins detection using the single particles counting technique.

The development of dark-field microscopic equipment greatly push much lower the detection limit of light scattering technique because of the greatly reduced background signals. One of the good evidence is the highly sensitive
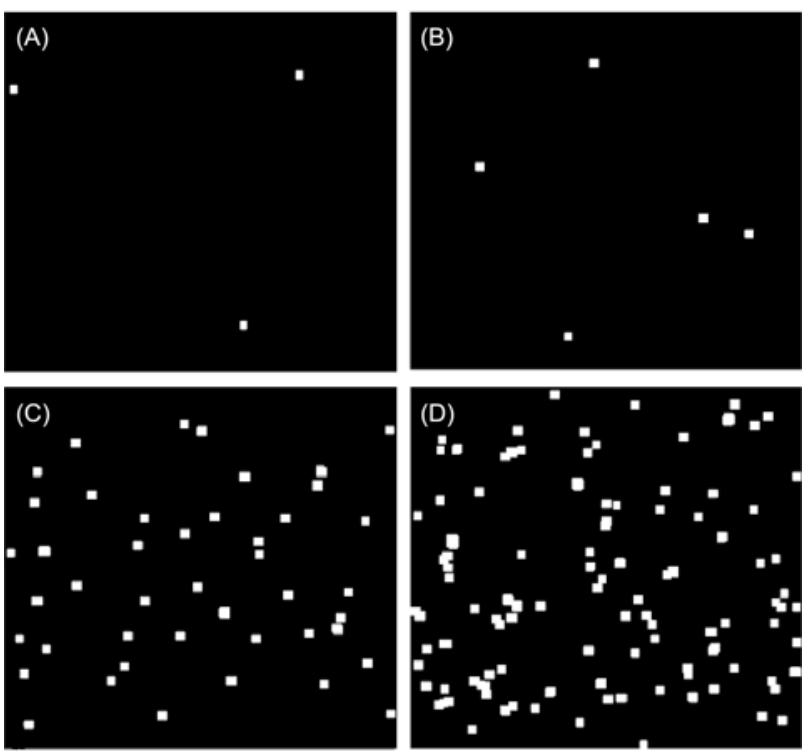

Figure 1 Single scattering particle counts of TPPS4 aggregation species induced by bovine serum albumin (BSA) at different concentrations. Concentration of TPPS4 is $0.1 \mu \mathrm{mol} / \mathrm{L}$ for (A), (C), and (D); no TPPS4 was added in (B). BSA concentrations (ng/mL): (A) 0; (B) 2000.0; (C) 60.0; (D) 180.0. The subframe area used for counting is $320 \times 280$ pixels $\left(\sim 0.02 \mathrm{~cm}^{2}\right)$. Threshold, 40; rank filters, 3. Reproduced with permission from Ref. [8], Copyright 2003 Elsevier. detection method of DNA as He groups [9] proposed by counting single scattering $\mathrm{Au}$ nanoparticles or their aggregates in free solution using flash-lamp dark-field microscopy. In the presence of target DNA, sandwich DNA hybridization induced mono-dispersed $\mathrm{Au}$ nanoparticles aggregated and the observed single scattering particle counts decreased under the dark-field microscope coupled with a flash-lamp illumination. By counting the scattering particles, the authors achieved a sensitive method for DNA detection with a limit of detection (LOD) of $0.1 \mathrm{pmol} / \mathrm{L}$.

For the single scattering particles counting technique, the sensitivity of detectors for single particles counting partly determines the detection sensitivity. Thus, development of highly sensitive instruments for counting single scattering particles is helpful to push the detection limit further lower, even down to the single molecular level. In this aspect, Yan groups [10] proposed a highly sensitive method using flow cytometer for size and concentration measurement of $\mathrm{Au}$ nanoparticles by detecting the scattering signals. More importantly, low concentration sample at about fmol/L level in a small volume ( $\mu \mathrm{L}$ level) can be measured within several minutes. Inspirited by this, by combining with HSFCM, the detection sensitivity of single scattering particles counting technique can be further improved.

\section{Single plasmonic nanoparticle sensing}

Plasmonic nanoparticles, especially $\mathrm{Au}$ and Ag nanoparticles, have gained great attention in the past 15 years. These nanoparticles have excellent light absorption and scattering properties, arising from the collective coherent oscillation of free electrons, termed as localized surface plasmon resonance (LSPR), when they expose to an electromagnetic field $[2,11,12]$. And LSPR is tuned by size, shape, surrounding medium, and plasmon coupling of nanoparticles [13-18]. Based on this, ensemble of resonant scattering nanoparticles has been used as LSPR sensors for bioassays [13,19-26]. Recently, single plasmonic nanoparticle based resonant scattering analytical technique using dark-field light scattering spectroscopy, which is the most straightforward mean for characterizing the LSPR spectra of single plasmonic nanoparticles, gains much attention. This technique makes darkfield images with a very low background, thus creating high signal-to-noise spectra of single nanoparticles. Furthermore, it has high spectral resolution, thus enables to reduce the absolute detection limit. By using single plasmonic nanoparticles, researchers developed dielectric environment change, plasmon coupling, and plasmon resonance energy transfer based sensing methods as well as chemical reaction recorders, as discussed below.

\subsection{Dielectric environment change}

Single plasmonic nanoparticles react to local dielectric 
nanoenvironment changes in their surroundings and show a shift of scattering spectrum [27,28]. Biochemical molecules always have higher refractive index (RI) than that of surrounding medium (always water) of plasmonic nanoparticles. Thus, their adsorption enables the local RI of single nanoparticles to increase, inducing the scattering spectrum to redshift. This phenomenon forms the principle of using single nanoparticles for biochemical sensing [29-45]. The LSPR light scattering spectral shift $\left(\Delta \lambda_{\text {max }}\right)$ induced by RI change, such as adsorbates can be expressed as

$$
\lambda_{\text {max }}=m\left(n_{\text {adsorbate }}-n_{\text {medium }}\right)\left(1-\mathrm{e}^{-2 d / l_{\mathrm{d}}}\right)[46],
$$

where $m$ represents the sensitivity factor and $l_{\mathrm{d}}$ is the decay length of electromagnetic field, both of which are determined by nanoparticle parameters, such as composition, size, and shape. $n_{\text {adsorbate }}$ is the RI of adsorbate, which has the effective thickness expressed as $d$. $n_{\text {medium }}$ is the RI of the medium surrounding the nanoparticles. For a given nanoparticle in water, $\Delta \lambda_{\max }$ is determined by $n_{\text {adsorbate }}$ and $d$ of adsorbates, in other words, by the molecular size of adsorbates. Thus, adsorption of biomolecules, such as proteins and DNAs will provide larger $\Delta \lambda_{\max }$ than small molecules.

For the sensing purpose, Van Duyne and McFarland [28] used single Ag nanoparticles as optical sensors for sensing the adsorption of thiols. Proof-of-concept experiment shows that the LSPR scattering spectrum of an Ag nanoparticle shifts to red direction as the RI of its dielectric environment increases, and the $\Delta \lambda_{\max }$ shows a good linearity with the RI. To further study the sensing ability of single Ag nanoparticles, the adsorption of 1-hexadecanthiol (1-HDT) was probed. Formation of a 1-HDT layer on a single Ag nanoparticle leads to a spectral shift of greater than $40 \mathrm{~nm}$, which corresponds to the detection of fewer than 60000 molecules of 1-HDT. Similarly, Raschke et al. [38] constructed such a typical single nanoparticle platform for detecting biomolecules. Biotinylated single $\mathrm{Au}$ nanospheres were used as plasmonic nanosensors, and specific binding of streptavidin to biotinylated single $\mathrm{Au}$ nanospheres changes their surrounding RI, which led to a redshift of scattering spectrum. The minimum detectable $\Delta \lambda_{\max }$ corresponds to about 1.0 $\mu \mathrm{mol} / \mathrm{L}$ of streptavidin. Thereafter, single Au nanorods and Au nanostars were employed for streptavidin detection using the same strategy with the detection limit of about 1.0 $\mathrm{nmol} / \mathrm{L}$ [30] and $0.1 \mathrm{nmol} / \mathrm{L}$ [29], respectively. These reports predict that the particle shape has great effect on the sensing ability of single plasmonic nanoparticle, which was further proven by investigating the shape dependent RI sensitivity of Au nanoparticles [18,47]. Additionally, this kind of single nanoparticle sensors can be used as molecular ruler for measuring the DNA length in nano scale and detecting the nuclease activity [42].

Such a single nanoparticle sensors can also be employed for sensing hydrogen, which, as well-known, is an explosive gas when its concentration exceeds $4 \%$. Thus, detection of hydrogen is a wide-concerned and vital safety issue. For this reason, Alivisatos groups [41] developed an $\mathrm{Au}$ antenna enhanced hydrogen sensing method using Pd nanoparticles at the single nanoparticle level. Pd nanoparticles can absorb and react with hydrogen to form Pd hydride, which changes their electrical and dielectric properties, in a reversible manner. This forms the basis of using Pd nanoparticles for hydrogen sensing. However, the scattering light of single Pd nanoparticle, barely visible, has very broad resonant Raleigh scattering spectrum. These properties block the using single Pd nanoparticles for hydrogen sensing. To address this problem, the authors employed Au nanoparticles as the enhanced hydrogen sensors because they scatter strongly in visible region and have marrow scattering band. Adsorption of hydrogen on Pd nanoparticle changes the dielectric environment of single $\mathrm{Au}$ nanoparticles and induces a shift in scattering spectrum for hydrogen sensing. Additionally, the authors found that the distance between Pd and Au antenna and the shape of $\mathrm{Au}$ antenna have great effect on the sensing capacity. Further work reported by them carefully studied the shape-dependent hydrogen uptake trajectories from individual $\mathrm{Au} / \mathrm{Pd}$ core-shell nanoparticles, monitoring by dark-field spectroscopy [32]. And an interesting find was that shape, faceting, and Pd shell thickness of Au nanoparticles play great role on hydrogen uptake trajectories. Furthermore, by using dark-field microscopy, the generation of hydrogen is monitoring by single nanoparticle probes [33].

In the most cases, single plasmonic nanoparticles sensing needs to scanning the scattering spectra of single nanoparticles with dark-field microscopy and spectroscopy, which are complicated to operate. To address this problem, we can develop new methods that can be extended and carried out in common laboratories. In this aspect, our group proposed a novel method of RGB analysis using individually colorcoded plasmonic Au nanoparticles [48], as shown in Figure 2. Scattering light colors of single $\mathrm{Au}$ nanoparticles were

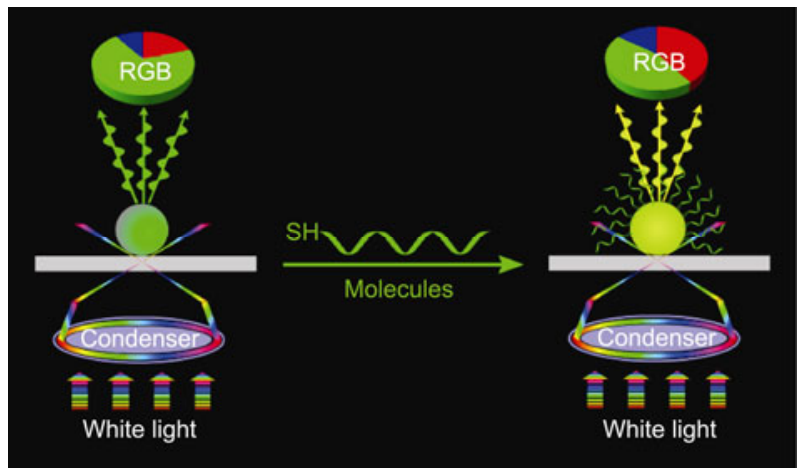

Figure 2 Scheme of the dark-field scattering imaging and RGB analysis for molecular binding studies based on the tricolor (RGB) system of an individual scattering color-coded plasmonic nanoparticle. After the binding of thiol molecules to a plasmonic nanoparticle, the RGB values of its scattering light color changes. This forms the basis of using single plasmonic nanoparticles for RGB analysis. Reprinted with permission from Ref. [48], Copyright 2011 Royal Society of Chemistry. 
coded with the tricolor (RGB) system by assigning digital values to $R, G$ and $B$. Knowledge is that the scattering light color of a plasmonic nanoparticle is RI dependent. Namely, the RGB values of single Au nanoparticles were RI dependent. Following proof-of-concept experiment showed that $R$ value increased and $G$ value decreased as the RI increases. Based on this, the binding of thiol molecules onto single $\mathrm{Au}$ nanoparticles was investigated through RGB analysis.

\subsection{Plasmon coupling}

When a plasmonic nanoparticle approaches in close to another one, their plasmonic oscillations couple together, termed as plasmon coupling. When plasmon coupling occurs, the scattering spectrum of a single nanoparticle shifts towards longer wavelength and shows a significant color change of scattering light $[42,49,50]$. In such case, He groups [51] developed a sensitive method for single molecule sensing through the scattering light color differentiation of single plasmonic nanoparticles. The authors synthesized spectrally uniform $\mathrm{Au}$ nanoparticles and $\mathrm{Au} / \mathrm{Ag} / \mathrm{Au}$ nanoparticles, which were then conjugated with two probe DNAs. In the presence of target DNA, sandwich hybridization pulled nanoparticles in close proximity to form dimers or oligomers. Plasmon coupling of nanoparticles induced color change of scattering light of single plasmonic nanoparticles, which can be observed using a conventional dark-field microscope combining with truecolor CCD camera. In contrast, disassembly of coupled nanoparticles shows a blue-shift of scattering light. Based on this, Lee groups [52] proposed a colorimetric method for biomolecules detection. Firstly, coresatellite $\mathrm{Au}$ nanoassemblies were prepared through biotinstreptavidin interaction with a peptide spacer of ten amino acids. After exposure of the assemblies to trypsin, peptides were cleaved and satellite $\mathrm{Au}$ nanoparticles disassembled apart from the surface of core Au nanoparticle. In such case, plasmon coupling between core and satellite Au nanoparticles was removed, resulting in the scattering light color change, which enables colorimetric detection of the protease.

Traditional Föster resonance energy transfer (FRET) is accepted by researchers and used to measure the interactions and conformational changes of molecules at the nano scale, always shorter than $10 \mathrm{~nm}$. Alternatively, plasmon coupling can be employed to develop long range molecular rulers because it is a function of particle separation [50,53-59]. In other words, the plasmon coupling strength that determines the magnitude of the spectral shift and intensity increase is distance dependent. More importantly, particle separations of about 2.5 times of nanoparticle diameter (always longer than $10 \mathrm{~nm}$ ) still lead to a measureable plasmon shift [53]. Thus, the plasmon coupling based molecular ruler has the advantage of longer detection range, and it can be employed as molecular rulers to break through the distance limit of FRET based molecular rulers.
Alivisatos groups [60] designed a plasmon ruler to investigate the dynamics of DNA hybridization by monitoring the distances between single $\mathrm{Au}$ or Ag nanoparticle pairs. At first, the authors constructed $\mathrm{Au}$ and $\mathrm{Ag}$ nanoparticle pairs through biotin-streptavidin interaction with a 33 bases DNA spacer (Figure 3(A)). Comparing with individual nanoparticles, $\mathrm{Au}$ and $\mathrm{Ag}$ nanoparticle pairs scatter stronger and longer wavelength lights under dark-field microscope due to plasmon coupling (Figure 3(B) and (C)). Correspondingly, the LSPR scattering spectra shift towards red direction (Figure 3(D)). And the spectral shift for Ag nanoparticles is $102 \mathrm{~nm}$, much larger than $23 \mathrm{~nm}$ of Au nanoparticles. This result indicates that Ag nanoparticles are preferred to be used for designing plasmon rulers. Then, the plasmon ruler was used to study the distance change induced by the ion strength of the incubation buffer. The plasmon resonance shifted to blue in buffer with low salt concentration $(0.005 \mathrm{~mol} / \mathrm{L})$, suggesting the increase of particles separation resulted from reduced electrostatic repulsion.
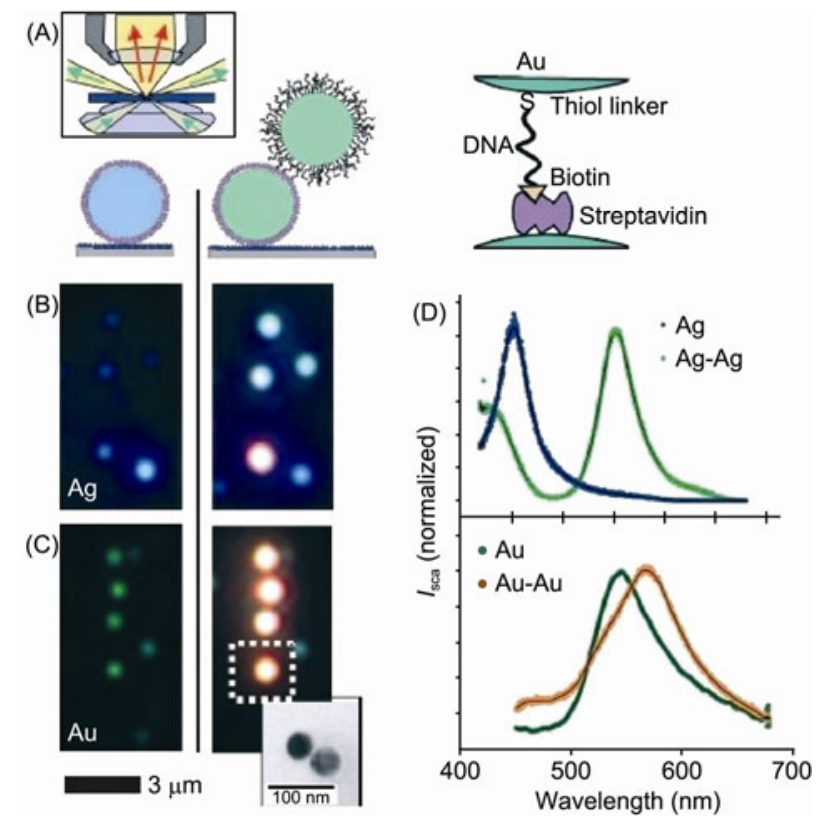

Figure 3 Color effect on directed assembly of DNA-functionalized $\mathrm{Au}$ and $\mathrm{Ag}$ nanoparticles. (A) First, nanoparticles functionalized with streptavidin were attached to a glass surface coated with BSA-biotin (left). Then, a second particle is attached to the first particle, again via biotin-streptavidin binding (right). The biotin on the second particle is covalently linked to the 3 ' end of a 33 base pair long ssDNA strand bound to the particle via a thiol group at the 5' end. Inset: principle of transmission DFM. (B) Single Ag particles appear blue (left) and particle pairs appear blue-green (right). The orange dot in the bottom comes from an aggregate of more than two particles. (C) Single Au particles appear green (left), Au particle pairs appear orange (right). Inset: representative transmission electron microscopy image of a particle pair to show that each colored dot comes from light scattered from two closely lying particles, which cannot be separated optically. (D) Representative scattering spectra of single particles and particle pairs for Ag (top) and Au (bottom). Ag particles show a larger spectral shift (102 $\mathrm{nm})$ than $\mathrm{Au}$ particles $(23 \mathrm{~nm})$, stronger light scattering and a smaller plasmon line width. Reproduced with permission from Ref. [60], Copyright 2005 Nature Publishing Group. 
When the salt concentration was restored to original value $(0.1 \mathrm{~mol} / \mathrm{L})$, redshift of scattering spectrum was observed. Furthermore, the nanoparticle pairs were used as molecular rulers for detecting DNA hybridization. Due to double strands DNA is much stiffer than single strand DNA, the nanoparticle pair was pushed apart and the particle separation increased after hybridization, and thus a significant blue-shift was observed. By monitoring the change of scattering spectrum of a single particle pair, the dynamic of DNA hybridization can be recorded in real-time. Thereafter, such nanoparticle pairs were served as plasmon rulers to study the kinetics of DNA bending and cleavage by restriction enzymes in vitro [61], and crown nanoparticles were used as plasmon ruler for light scattering imaging of caspase-3 activation at early-stage in living cells [62].

\subsection{Plasmon resonance energy transfer (PRET)}

In 2007, Lee groups [63] observed the plasmon resonance energy transfer (PRET) for the first time from single plasmonic nanoparticles (donors) to its surface adsorbed biomolecules (acceptors). Similar with Föster resonance energy transfer (FRET), PRET occurs when the LSPR light scattering spectrum of the donor nanoparticle overlaps with the absorption spectra of the adsorbed molecules. Although the mechanism of PRET is not clearly understood up to now, it seems that the energy (scattering light) transfers through the interaction between the plasmon resonant dipole of the plasmonic nanoparticle and the molecular dipole of the adsorbed molecules.

When PRET occurs, the LSPR scattering light of a single nanoparticle is quenched and shows a spectral quenching dip [63] or decrease of scattering intensity [64,65] determined by the absorption spectral broadness of the energy acceptor molecules. Cytochrome $c$, a metalloprotein, has narrow absorption band of both reduced (central at 525 and $550 \mathrm{~nm}$ ) and oxidized (central at $530 \mathrm{~nm}$ ) state, overlapping with the LSPR light scattering spectrum of a $30 \mathrm{~nm}$ Au nanoparticle ranging from 530 to $580 \mathrm{~nm}$ (Figure 4(A) and (D)). When reduced cytochrome $c$ molecules coupled to a $30 \mathrm{~nm}$ Au nanoparticle surface, distinct resonant quenching dips at about 525 and $550 \mathrm{~nm}$ were observed in its scattering spectrum due to PRET (Figure 4(B) and (E)). As the same, resonant quenching dips at about $530 \mathrm{~nm}$ was observed in the scattering spectrum after oxidized cytochrome $c$ was coupled to single Au nanoparticles (Figure 4(C) and 4(F)). While no scattering quenching dips were observed from control experiments: peptide coated $30 \mathrm{~nm} \mathrm{Au}$ nanoparticles (Figure 4(G)), cytochrome $c$ coated large Au nanoparticles (Figure 4(H)), cytochrome $c$ coated polystyrene nanoparticle (Figure 4(I)). These results indicated that the requirements of the spectral overlapping and the presence of excited free electrons are necessary for the occurrence of PRET.

Thereafter, PRET-based spectroscopic imaging method was developed for monitoring the fluctuations of intracellular cytochrome $c$ in real time in living cells by exploiting quenching dips which results from the selective energy transfer from single Au nanoparticle to cytochrome $c$ [66]. As the same, researchers developed PRET based single nanoparticle sensors for quantitative detection of metal ion [64] and explosives [65]. This method offers high spatial resolution, provides high sensitivity, and shows high selectivity for analytical purposes. Generally, this detection mode is based on the coordination chemistry of targets with their ligands. For example, Lee groups [65] used a $\mathrm{Cu}^{2+}$ ligand, which has no absorption band overlapping with the resonant scattering band of $50 \mathrm{~nm}$ Au nanoparticles. Thus, no PRET was observed when the ligands were coupled to Au nanoparticles. While, exposure of the ligand modified single $\mathrm{Au}$ nanoparticles to $\mathrm{Cu}^{2+}$, PRET occurred and showed a decrease of scattering intensity resulted from the formation of $\mathrm{Cu}^{2+}$-ligands complex, which has broad adsorption band overlapping with the scattering spectra of $50 \mathrm{~nm}$ AuNPs. Similarly, cysteine modified Au nanoparticles were used as single PRET based nanosensors for 2,4,6-trinitrotoluene (TNT) detection.

\subsection{Chemical reaction recorders}

Metabolism in organisms involves in many biochemical reactions, which are critical for energy exchange and drugs screening. However, in-situ measurement of biochemical reaction remains a fundamental challenge for most researchers nowadays. LSPR spectrum of a single plasmonic nanoparticle is sensitive to its surrounding changes, such as electron injection and metal atoms deposition. Thus, we can clearly understand the chemical reaction by monitoring the scattering spectrum of a single plasmonic nanoparticle. As reported by Mulvaney groups [67], the electron injection and extraction of an Au nanorod were monitored during a redox reaction of ascorbic acid by dissolved oxygen on its surface and the kinetics of atomic deposition onto a single Au nanorod were further studied. As the reaction time prolongs, the spectra shifted blue of about $20 \mathrm{~nm}$ in the first $3 \mathrm{~min}$, indicating the electrons are injected. Then, it reached a plateau, suggesting that the Au nanorod had the highest electron density. Thereafter, the light scattering spectra of the single Au nanorod red-shifted back to the initial value. And the calculated electron injected rate was 4600 electrons per second.

Then, single Au nanorods were used to observe the deposition of $\mathrm{Au}$ atoms generating from chemical reaction by monitoring the changes of resonant Raleigh scattering spectrum. The deposition of $\mathrm{Au}$ atoms onto the Au nanorod tips, the lowest energy surfaces, induces the aspect ratio of single $\mathrm{Au}$ nanorods to increase and thus shows a redshift of scattering spectrum. As shown in Figure 5(A), after exposure of $\mathrm{Au}$ nanorods to the grow solution containing $\mathrm{CTAB}, \mathrm{HAuCl}_{4}$ and ascorbic acid, the scattering spectrum of a single $\mathrm{Au}$ 

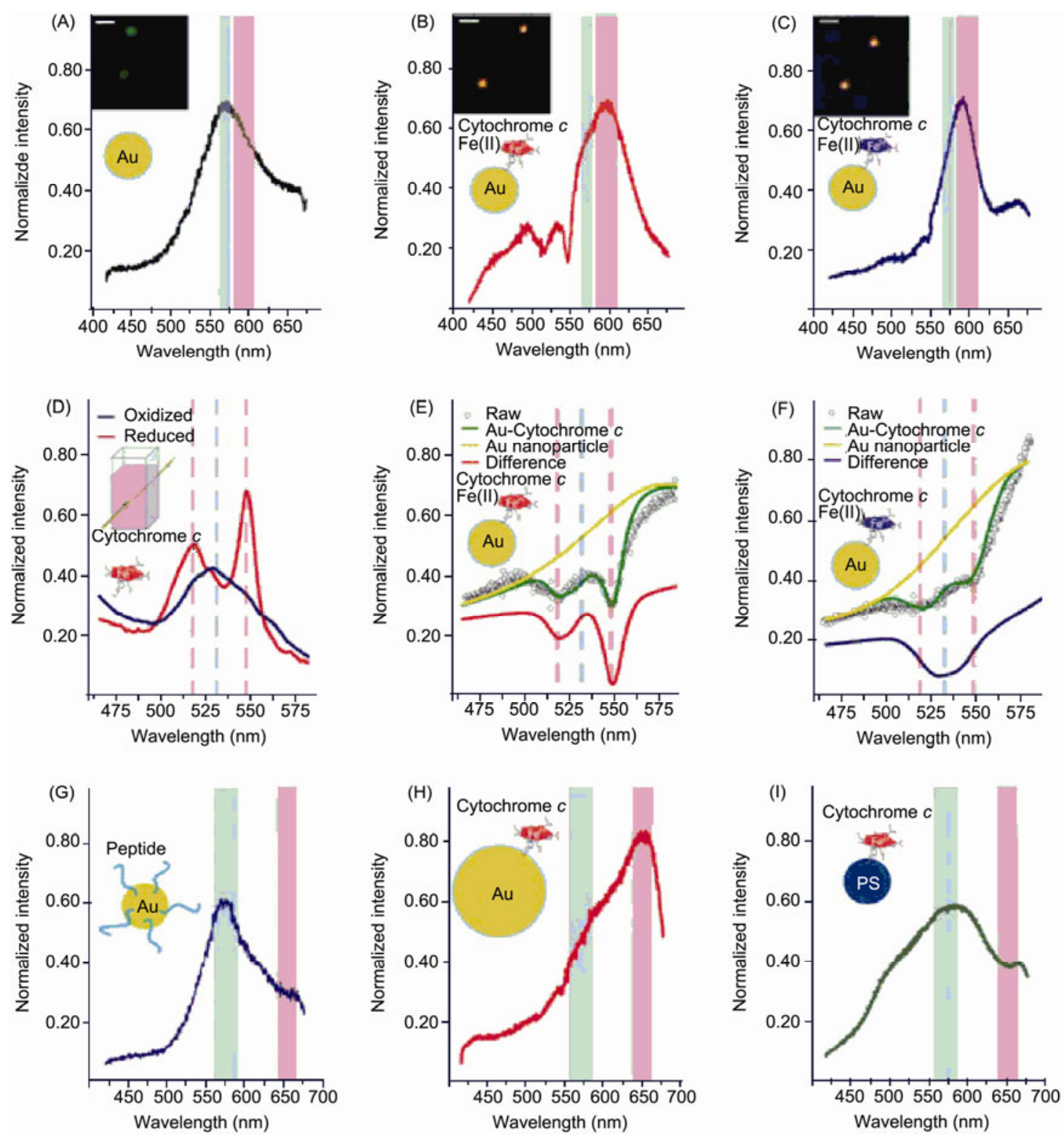

Figure 4 Experimental results of PRET from single AuNP to conjugated cytochrome c molecules. (A-C) The Rayleigh scattering spectra of three $30 \mathrm{~nm}$ AuNPs coated with (A) cysteamine only, (B) cysteamine and reduced cytochrome $c$, and (C) cysteamine and oxidized cytochrome $c$. Insets: truecolor scattering images of individual nanoparticles. Scale bars, $2 \mathrm{~mm}$. (D) The bulk visible absorption spectra of oxidized (blue solid line) and reduced (red solid line) $8 \mathrm{mmol} / \mathrm{L}$ cytochrome $c$ using conventional UV-vis absorption spectroscopy. Dashed lines were drawn to facilitate identification of peak wavelengths. (E) Fitting curve for the spectrum in (B). Green solid line, fitting curve of the raw data; yellow solid line, Lorentzian scattering curve of bare Au nanoparticle; red solid line, processed absorption spectra for reduced conjugated cytochrome $c$ (green minus yellow curve). (F) The fitting curve for the spectrum in (C). Blue solid line, processed absorption spectra for oxidized conjugated cytochrome $c$ (green minus yellow curve). (G-I) PRET spectra of (G) Au nanoparticle coated with Cys-(Gly-Hyp-Pro) 6 peptide, (H) cytochrome $c$ on large Au nanoparticle, and (I) cytochrome $c$ on a $40 \mathrm{~nm}$ polystyrene nanoparticle. Reproduced with permission from Ref. [63], Copyright 2007 Nature Publishing Group.

nanorod shifted towards red direction as the time get longer, which can also see from Figure 5(B). While the control experiment show no spectral shift. Using DDA for calculation, the $\mathrm{Au}$ nanorod has aspect ratio of 2.87 (Figure 5(C)), which changed to 3.01 after the deposition of Au atoms on its tips. Thus, the redshift in scattering spectrum is resulted from the gradual increase of aspect ratio of Au nanorod. The relationship between plasmon band maximum and aspect ratio (Figure 5(D)) further approves the above conclu- sion. The increase in aspect ratio corresponds to the deposition of $\sim 50000$ atoms for an 8 min reaction time or just $\sim 100$ atoms of Au every second.

Similarly, Long groups [68] used single Au nanoparticles for the real-time detection of NADH-dependent intracellular metabolic enzymatic pathways, which is an important processes and plays an critical role in many metabolisms of organism. Au catalyzed reduction of $\mathrm{Cu}^{2+}$ ions by NADH generates $\mathrm{Cu}^{0}$, which then deposited onto $\mathrm{Au}$ nanoparticles 

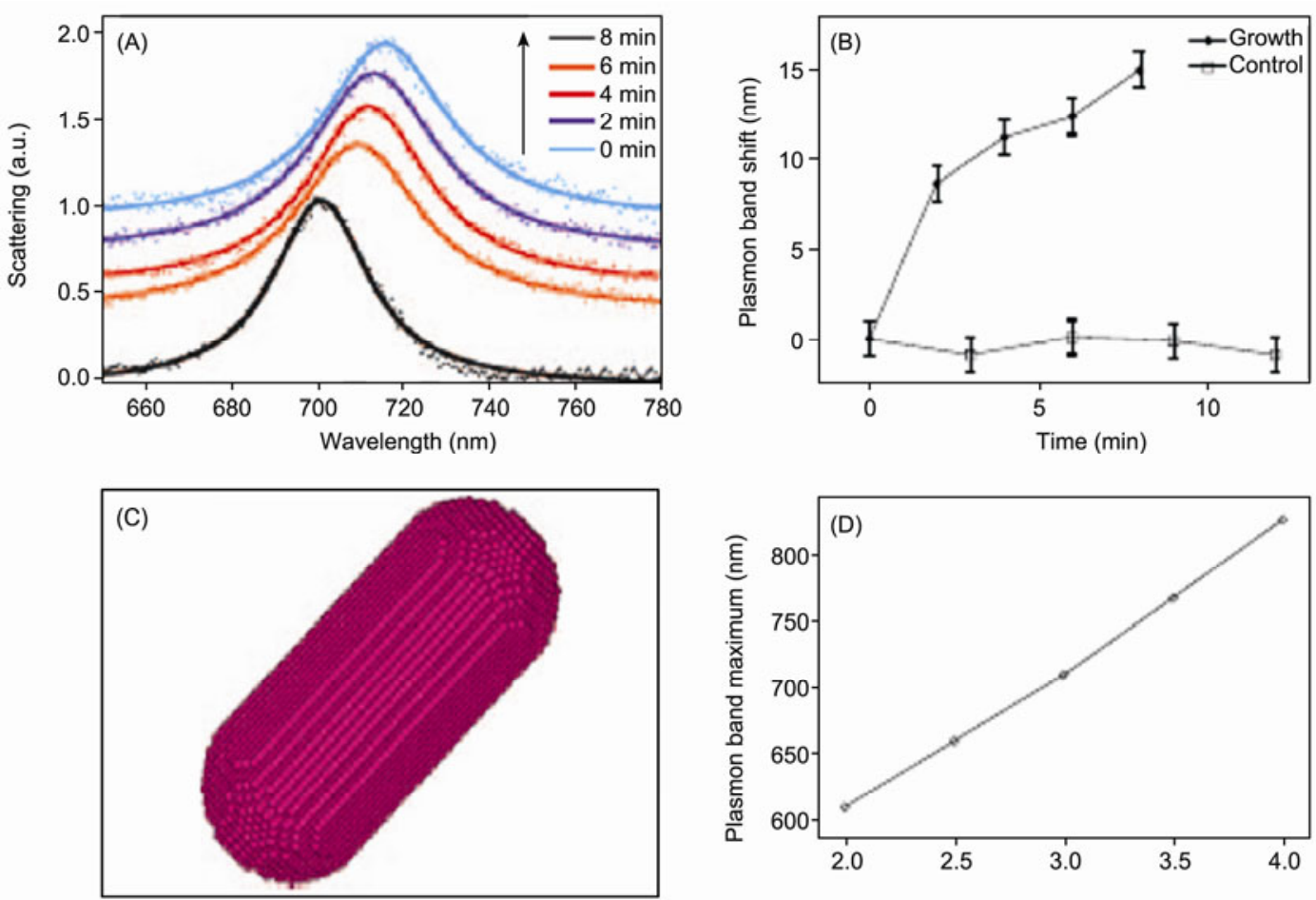

Figure 5 Growth of a single Au nanorod. (A) Evolution of the scattering spectrum of an Au nanorod (aspect ratio 2.87) after a growth solution containing $\mathrm{CTAB}, \mathrm{HAuCl}_{4}$ and ascorbic acid (reducing agent) is added. Spectra are normalized and the full lines are the Lorentzian fits to the experimental data. (B) SP band shift as a function of time for the growth experiment and for a control experiment. The error bars represent the error in determining the peak position from the Lorentzian fitting procedure. (C) Typical discrete dipole approximation (DDA) target constructed to simulate hemispherically capped Au nanorods containing 52000 dipoles and aspect ratio of 2.87. (D) Predicted SP position versus aspect ratio for hemispherically capped rods in a medium with relative permittivity $\varepsilon_{\mathrm{m}}=2.025$ based on DDA results. Reproduced with permission from Ref. [67], Copyright 2008 Nature Publishing Group.

to form $\mathrm{Au} / \mathrm{Cu}$ core-shell nanostructures. These core-shell nanoparticles have longer scattering bands comparing with single bare Au nanoparticles. And in the absence of NADH, no spectral shift was observed. The magnitude of spectral shift is reaction time and NADH concentration dependent. As the reaction time prolongs or the NADH concentration increases, the spectrum shifts to longer wavelength. Then, it was extended to investigate the metabolism in cancer cells, and especially, the effect of anticancer drugs on the cell metabolism was studied. In addition, single nanoparticle spectroscopy can be used to study the oxidation of single $\mathrm{Cu}$ nanoparticles [69].

\section{Single plasmonic nanoparticle tracking}

Quantum dots (QDs), the mostly used nanoparticles for tracking the dynamic events in vivo, attract great attention in recent years [70,71]. However, QDs always suffer from the disadvantages of high cytotoxicity, hard modification. With the development of nanotechnology, plasmonic nanoparticles are gradually accepted by researchers and used as optical probes for imaging because they possess the following advantages. (1) They scatter strong light. For a $60 \mathrm{~nm} \mathrm{Au}$ nanoparticle, the molar extinction coefficient $(\varepsilon)$ is $6.33 \times$
$10^{-11} \mathrm{~L} /(\mathrm{mol} \mathrm{cm})$ and the light scattering yield $\left(\varphi_{\mathrm{s}}\right)$ is 0.313 [2]. Thus, the light scattering power equals to $\varepsilon \varphi_{\mathrm{s}}=$ $1.98 \times 10^{-11} \mathrm{~L} /(\mathrm{mol} \mathrm{cm})$, five orders of magnitude higher than the light producing power of fluorescein. (2) The scattering light is tunable in the UV-visible-infrared region. Light wavelength scattering from metal nanoparticles is determined by their compositions, sizes, shapes, and surrounding mediums. For example, $40 \mathrm{~nm} \mathrm{Ag} \mathrm{nanosphere}$ scatters blue light; $50 \mathrm{~nm}$ Au nanosphere scatters green light; and $100 \mathrm{~nm} \mathrm{Ag} \mathrm{triangular} \mathrm{prism} \mathrm{scatters} \mathrm{red} \mathrm{[72].} \mathrm{Thus,} \mathrm{we}$ can synthesize nanoparticles with rainbow scattering light by controlling their sizes and shape [15,73]. (3) Their scattering lights are stable and anti-photo bleaching, which enables long-term imaging. (4) They have low cytotoxicity, compared with QD. (5) They are facile to be synthesized and modified with biochemical molecules, such as DNA and proteins. These properties enable metal nanoparticles to be nice candidates as optical probes for cell/tissues imaging [74-78].

Nam and Yang [79] developed a method for detecting the receptor-ligand interaction by tracking the scattering signals of single $\mathrm{Au}$ nanoparticles. In this work, the authors constructed a cell membrane mimicking lipid bilayer on glass slide for cholera toxin detection by tracking the trajectories of single membrane bound $\mathrm{Au}$ nanoparticles using dark- 
field microscopy. In the presence of cholera toxin, the diffusion coefficients of single membrane bound Au nanoparticles were reduced resulted from the decreased fluidity of lipid bilayer due to the specific interaction of cholera toxin with ganglioside $\mathrm{GM}_{1}$ in the membrane. This forms the basis of receptor-ligand interactions detection through single nanoparticle tracking. And by increasing the mobility of lipid tethered $\mathrm{Au}$ nanoparticles, the sensitivity can be further improved. Further find was that paucivalent Au nanoparticles show higher diffusion coefficients than that of multivalent $\mathrm{Au}$ nanoparticles on the lipid bilayer. The detection limit for cholera toxin is $10-100 \mathrm{pmol} / \mathrm{L},>100$-fold higher than fluorescence signal-based detection methods.

By tracking the scattering spectra of single nanoparticles, one can get the location and nanoenvironment information of single nanoparticles in living cells, which may help to know the intracellular pathways of single nanoparticles and for further biomedical imaging and disease diagnosis. For this reason, Asahi groups [80] developed a confocal Rayleigh scattering spectroscopic imaging system to real-time tracking the spectra and spatial positions of single Au nanoparticles to reveal their interaction with living mouse fibroblast cells in vivo. Due to the difference of RI of the local environment out of or in cells and the plasmon resonance peak wavelength of an Au nanoparticle is sensitive to its local environment, the Rayleigh scattering spectra of a single Au nanoparticle showed rapid spectral changes when they enter and then leave the vicinity of cell organelles and membranes. These variations constitute the basis of using single nanoparticles for tracking their interaction with and transportation in cells. Combining with a confocal Rayleigh scattering spectroscopic imaging system, the authors can observe the intracellular movement of a typical $\sim 80 \mathrm{~nm}$ nanoparticle in a fibroblast cell. And the recorded scattering spectral maximum wavelength of the nanoparticle further reflects the changes in the cell local environment and demonstrates the interaction mechanism of single nanoparticles with cells. Observation was that scattering maximum wavelength shifted to the red of $12 \mathrm{~nm}$ along with a decrease of the depth of the particle at $28 \mathrm{~min}$, followed by a return to its initial state after $5 \mathrm{~min}$. This can be explained by one of the tracking case: an increase in the RI of local environment after the particle penetrates in the membrane of the cell (or early endosome), because it may be closely packed by a lipid double layer.

In addition, our group [81] developed a novel single nanoparticle probes for intracellular prion protein imaging and single nanoparticle spectral analysis at the same time, as displayed in Figure 6. Aptamer that specifically recognizes prion protein was conjugated to Ag nanoparticles through streptavidin-biotin chemistry. The prepared optical probes were stable and biocompatible, which were then used for intracellular prion protein imaging and spectral analysis. After incubation of the conjugates with human bone marrow neuroblastoma (SK-N-SH) cells, scattering light from single $\mathrm{Ag}$ nanoparticles in living cells could be clearly observed
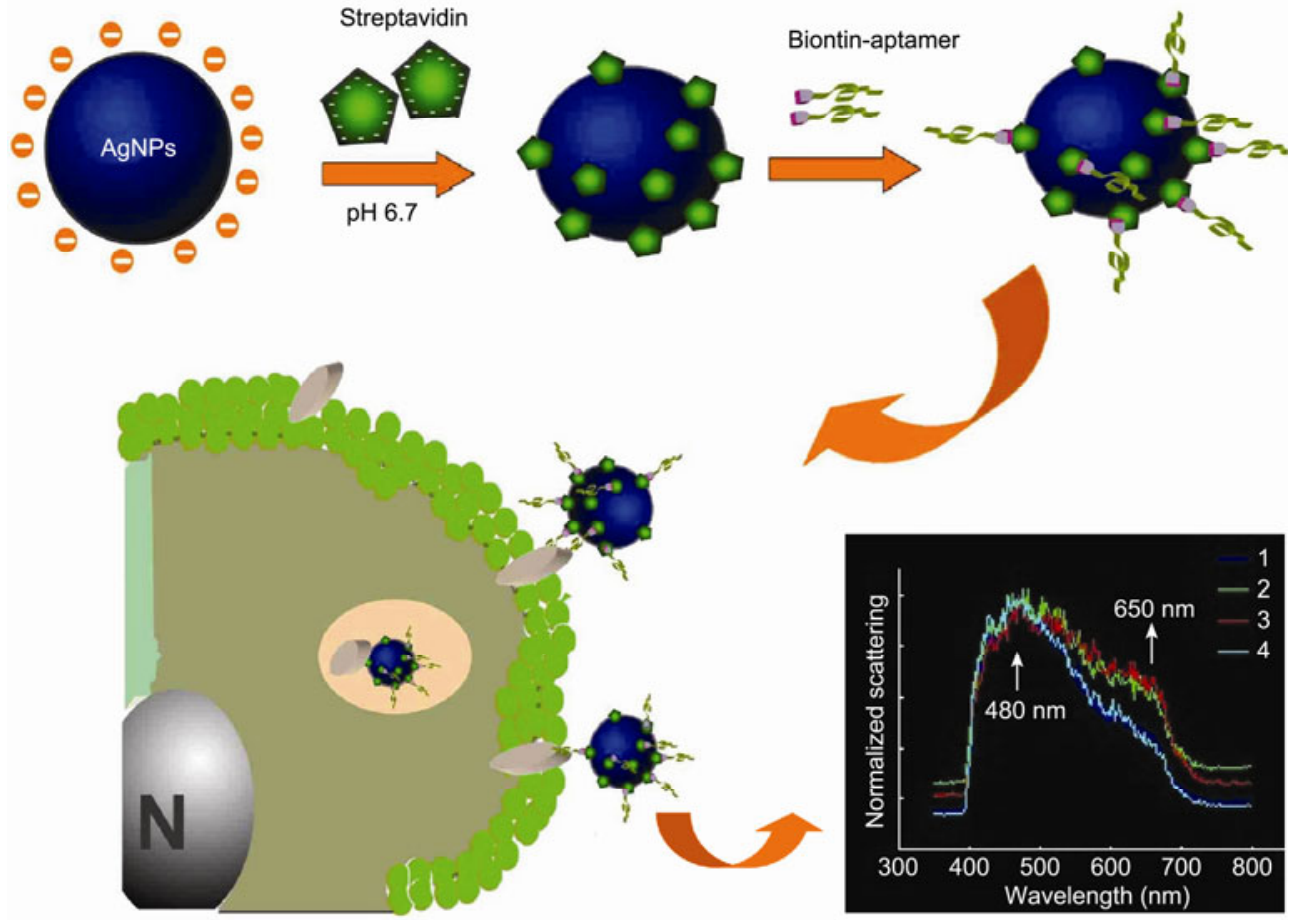

Figure 6 Schematic illustration of apt-Ag nanoparticle probes for simultaneous intracellular protein imaging and single nanoparticle spectral analysis. Aptamer conjugated Ag nanoparticle were prepared through streptavidin-biotin chemistry. Then, the aptamer conjugated Ag nanoparticles were applied as biocompatible probes for imaging prion protein in SK-N-SH cells. The scattering spectra of single Ag nanoparticles that internalized the cell are shown. Reproduced with permission from Ref. [81], Copyright 2010 American Chemical Society. 
using a common dark-field microscope. Negative controls by incubating of ploy $\mathrm{T}_{17}$ and streptavidin conjugated $\mathrm{Ag}$ nanoparticles with live cells under the same conditions showed that there was no significant number of bound $\mathrm{Ag}$ nanoparticles in the cells. These results told that our prepared optical probes could specifically target prion protein in living cells. Further single nanoparticles spectral analysis suggested that the potential use of plasmonic nanoparticles as nanosensors for single nanoparticle sensing in living cells. Finally, the intracellular pathway of aptamer conjugated Ag nanoparticles was investigated using TEM imaging. The results indicated that the caveolae-related endocytosis is likely a necessary pathway for the internalization of aptamer$\mathrm{Ag}$ nanoparticles in SK-N-SH cells.

\section{Summary and outlook}

In summary, this mini review summarizes the single scattering particles based analytical techniques, including single scattering particles counting, single plasmonic nanoparticle sensing, and single plasmonic nanoparticle tracking. And our focus is emphasized on the discussion of the techniques combining with dark-field microscopy and resonant Rayleigh scattering spectroscopy, such as single RI change based sensing platforms, plasmon coupling based molecular rulers, PRET based single nanosensors, and LSPR based biochemical reaction recorders. In the near future, the research focus of single scattering particles based analytical techniques, in our opinion, will continuously concern with the following aspects.

(1) Screening highly sensitive single nanoparticle sensing units with high spectral resolution by constructing light scattering imaging tree, similar with mass spectral trees [82]. Well-known is that shape has great effect on the RI sensitivity. Thus, to push the detection limit further lower, nanoparticles of new structures with higher aspect ratio and shaper corner can be synthesized and used as single sensing units. In addition, improvement of the sensitivity of the optical detector, the sensitivity of single particle nanosensors can be further improved.

(2) Design of plasmon coupling based long range molecular rulers. This kind of molecular rulers breaks through the distance limit, always shorter than $10 \mathrm{~nm}$, of traditional FRET based molecular rulers. Thus, plasmon molecular rulers can be widely used for measuring the molecular interactions and conformational changes in long range from several $\mathrm{nm}$ to hundreds of $\mathrm{nm}$.

(3) Study of PRET. On one hand, research concern will focus on the design of PRET based single nanoparticle sensors for sensitive and selective detection of biochemical molecules in vivo. On the other hand, as a new energy transfer form, to the best of our knowledge, only four reports were published on this topic up to now. But the theory about PRET, such as the distance and orientation effect on the efficiency of PRET, is not fulfilled, and to clearly understand PRET, careful study should be made in the near future.

(4) Real-time monitoring biochemical reaction in vivo. Biochemical reactions play a critical role on the metabolism, energy generation and exchange in the biosystem. By realtime monitoring the scattering feature changes of single plasmonic nanoparticles in vivo, we can learn the critical biochemical reaction in biosystem, which may in turn provide a new opportunity for drug screening, disease diagnosis, and cancer therapy.

(5) Real-time tracking dynamic events using single plasmonic nanoparticles in vivo. Presently, QDs are the mostly used optical probes for single particle tracking in vivo. However, the disadvantages from them make us need to search for better optical probes. In this aspect, plasmonic nanomaterial is a nice choice. In addition, the light source for dark-field light scattering imaging is so weak to damage live cells during long time imaging. Plus their excellent scattering property, it is reasonable to believe that plasmonic nanoparticles used as light scattering probes for imaging will become another research focus.

This work was supported by the National Natural Science Foundation of China (21035005), the Ministry of Science and Technology of the People's Republic of China (2011CB933600) and the Postgraduate Science and Technology Innovation Program of Southwest China University (ky2011006).

1 Pasternack R F, Collings P J. Resoanance light scattering: A new technique for studying chrommophore aggregation. Science, 1995, 269: 935-939

2 Yguerabide J, Yguerabide E E. Light-scattering submicroscopic particles as highly fluorescent analogs and their use as tracer labels in clinical and biological applications I. Theory. Anal Biochem, 1998, 262: 137-156

3 Huang C Z, Li K A, Tong S Y. Determination of nucleic acids by a resonance light-scattering technique with $\alpha, \beta, \gamma, \delta$-tetrakis[4-(trimethylammoniumyl)phenyl]porphine. Anal Chem, 1996, 68: 2259-2263

4 Huang C Z, Li K A, Tong S Y. Determination of nanograms of nucleic acids by their enhancement effect on the resonance light scattering of the cobalt(II)/4-[(5-chloro-2-pyridyl)azo]-1,3-diaminobenzene complex. Anal Chem, 1997, 69: 514-520

5 Elghanian R, Storhoff J J, Mucic R C, et al. Selective colorimetric detection of polynucleotides based on the distance-dependent optical properties of gold nanoparticles. Science, 1997, 277: 1078-1081

6 Storhoff J J, Elghanian R, Mucic R C, et al. One-pot colorimetric differentiation of polynucleotides with single base imperfections using gold nanoparticle probes. J Am Chem Soc, 1998, 120: 1959-1964

7 Taton T A, Lu G, Mirkin C A. Two-color labeling of oligonucleotide arrays via size-selective scattering of nanoparticle probes. J Am Chem Soc, 2001, 123: 5164-5165

8 Huang $\mathrm{C} \mathrm{Z}$, Liu Y, Wang Y H, et al. Resonance light scattering imaging detection of proteins with $\alpha, \beta, \gamma, \delta$-tetrakis ( $p$-sulfophenyl) porphyrin. Anal Biochem, 2003, 321: 236-243

9 Yuan Z, Cheng J, Cheng X, et al. Highly sensitive DNA hybridization detection with single nanoparticle flash-lamp darkfield microscopy. Analyst, 2012, 137: 2930-2932

10 Zhu S, Yang L, Long Y, et al. Size differentiation and absolute quantification of gold nanoparticles via single particle detection with a laboratory-built high-sensitivity flow cytometer. J Am Chem Soc, 2010, 132: $12176-12178$ 
11 Yguerabide J, Yguerabide E E. Light-scattering submicroscopic particles as highly fluorescent analogs and their use as tracer labels in clinical and biological applications II. Experimental characterization. Anal Biochem, 1998, 262: 157-176

12 Willets K A, Van Duyne R P. Localized surface plasmon resonance spectroscopy and sensing. Annu Rev Phys Chem, 2007, 58: 267-297

13 Ling J, Li Y F, Huang C Z. Visual sandwich immunoassay system on the basis of plasmon resonance scattering signals of silver nanoparticles. Anal Chem, 2009, 81: 1707-1714

14 Ringe E, McMahon J M, Sohn K, et al. Unraveling the effects of size, composition, and substrate on the localized surface plasmon resonance frequencies of gold and silver nanocubes: A systematic single-particle approach. J Phys Chem C, 2010, 114: 12511-12516

15 Mock J J, Barbic M, Smith D R, et al. Shape effects in plasmon resonance of individual colloidal silver nanoparticles. J Chem Phys, 2002, 116: 6755-6759

16 Lee K S, El-Sayed M A. Gold and silver nanoparticles in sensing and imaging: Sensitivity of plasmon response to size, shape, and metal composition. J Phys Chem B, 2006, 110: 19220-19225

17 Miller M M, Lazarides A A. Sensitivity of metal nanoparticle surface plasmon resonance to the dielectric environment. J Phys Chem B, 2005, 109: 21556-21565

18 Chen H, Shao L, Woo K C, et al. Shape-dependent refractive index sensitivities of gold nanocrystals with the same plasmon resonance wavelength. J Phys Chem C, 2009, 113: 17691-17697

19 He W, Huang C Z, Li Y F, et al. One-step label-free optical genosensing system for sequence-specific DNA related to the human immunodeficiency virus based on the measurements of light scattering signals of gold nanorods. Anal Chem, 2008, 80: 8424-8430

20 Liu Y, Huang C Z. One-step conjugation chemistry of DNA with highly scattered silver nanoparticles for sandwich detection of DNA. Analyst, 2012, 137: 3434-3436

21 Qi W J, Wu D, Ling J, et al. Visual and light scattering spectrometric detections of melamine with polythymine-stabilized gold nanoparticles through specific triple hydrogen-bonding recognition. Chem Commun, 2010, 46: 4893-4895

22 Liu Z D, Li Y F, Ling J, et al. A localized surface plasmon resonance light-scattering assay of mercury(II) on the basis of $\mathrm{Hg}^{2+}$-DNA complex induced aggregation of gold nanoparticles. Environ Sci Technol, 2009, 43: 5022-5027

23 Du B A, Li Z P, Liu C H. One-step homogeneous detection of DNA hybridization with gold nanoparticle probes by using a linear light-scattering technique. Angew Chem Int Ed, 2006, 45: 8022-8025

24 Su X, Kanjanawarut R. Control of metal nanoparticles aggregation and dispersion by pna and pna-DNA complexes, and its application for colorimetric DNA detection. ACS Nano, 2009, 3: 2751-2759

25 Tan Y N, Su X, Liu E T, et al. Gold-nanoparticle-based assay for instantaneous detection of nuclear hormone receptor-response elements interactions. Anal Chem, 2010, 82: 2759-2765

26 Tan Y N, Su X, Zhu Y, et al. Sensing of transcription factor through controlled-assembly of metal nanoparticles modified with segmented DNA elements. ACS Nano, 2010, 4: 5101-5110

27 Mock J J, Smith D R, Schultz S. Local refractive index dependence of plasmon resonance spectra from individual nanoparticles. Nano Lett, 2003, 3: 485-491

28 McFarland A D, Van Duyne R P. Single silver nanoparticles as realtime optical sensors with zeptomole sensitivity. Nano Lett, 2003, 3: 1057-1062

29 Dondapati S K, Sau T K, Hrelescu C, et al. Label-free biosensing based on single gold nanostars as plasmonic transducers. ACS Nano, 2010, 4: 6318-6322

30 Nusz G J, Marinakos S M, Curry A C, et al. Label-free plasmonic detection of biomolecular binding by a single gold nanorod. Anal Chem, 2008, 80: 984-989

31 Guo L, Kim D H. Reusable plasmonic aptasensors: Using a single nanoparticle to establish a calibration curve and to detect analytes. Chem Commun, 2011, 47: 7125-7127

32 Tang M L, Liu N, Dionne J A, et al. Observations of shape-dependent hydrogen uptake trajectories from single nanocrystals. J Am Chem
Soc, 2011, 133: 13220-13223

33 Seo D, Park G, Song H. Plasmonic monitoring of catalytic hydrogen generation by a single nanoparticle probe. J Am Chem Soc, 2011, 134: $1221-1227$

34 Cao C, Sim S J. Resonant rayleigh light scattering response of individual au nanoparticles to antigen-antibody interaction. Lab Chip, 2009, 9: 1836-1839

35 Ament I, Prasad J, Henkel A, et al. Single unlabeled protein detection on individual plasmonic nanoparticles. Nano Lett, 2012, 12: 10921095

36 Baciu C L, Becker J, Janshoff A, et al. Protein-membrane interaction probed by single plasmonic nanoparticles. Nano Lett, 2008, 8: 17241728

37 Raschke G, Brogl S, Susha A S, et al. Gold nanoshells improve single nanoparticle molecular sensors. Nano Lett, 2004, 4: 1853-1857

38 Raschke G, Kowarik S, Franzl T, et al. Biomolecular recognition based on single gold nanoparticle light scattering. Nano Lett, 2003, 3 : 935-938

39 Rindzevicius T, Alaverdyan Y, Dahlin A, et al. Plasmonic sensing characteristics of single nanometric holes. Nano Lett, 2005, 5: 2335-2339

40 Song H D, Choi I, Yang Y I, et al. Picomolar selective detection of mercuric ion $\left(\mathrm{Hg}^{2+}\right)$ using a functionalized single plasmonic gold nanoparticle. Nanotechnology, 2010, 21: 145501

41 Liu N, Tang M L, Hentschel M, et al. Nanoantenna-enhanced gas sensing in a single tailored nanofocus. Nat Mater, 2011, 10: 631-636

42 Liu G L, Yin Y, Kunchakarra S, et al. A nanoplasmonic molecular ruler for measuring nuclease activity and DNA footprinting. Nat Nanotechnol, 2006, 1: 47-52

43 Guo L, Ferhan A R, Lee K, et al. Nanoarray-based biomolecular detection using individual au nanoparticles with minimized localized surface plasmon resonance variations. Anal Chem, 2011, 83: 26052612

44 Sannomiya T, Hafner C, Voros J. Shape-dependent sensitivity of single plasmonic nanoparticles for biosensing. J Biomed Optics, 2009, 14: 064027

45 Sannomiya T, Voros J. Single plasmonic nanoparticles for biosensing. Trends Biotechnol, 2011, 29: 343-351

46 Anker J N, Hall W P, Lyandres O, et al. Biosensing with plasmonic nanosensors. Nat Mater, 2008, 7: 442-453

47 Chen H, Kou X, Yang Z, et al. Shape- and size-dependent refractive index sensitivity of gold nanoparticles. Langmuir, 2008, 24: 5233-5237

48 Liu Y, Ling J, Huang C Z. Individually color-coded plasmonic nanoparticles for RGB analysis. Chem Commun, 2011, 47: 8121-8123

49 Sannomiya T, Hafner C, Voros J. In situ sensing of single binding events by localized surface plasmon resonance. Nano Lett, 2008, 8: 3450-3455

50 Reinhard B M, Siu M, Agarwal H, et al. Calibration of dynamic molecular rulers based on plasmon coupling between gold nanoparticles. Nano Lett, 2005, 5: 2246-2252

51 Xiao L, Wei L, He Y, et al. Single molecule biosensing using color coded plasmon resonant metal nanoparticles. Anal Chem, 2010, 82: 6308-6314

52 Waldeisen J R, Wang T, Ross B M, et al. Disassembly of a core-satellite nanoassembled substrate for colorimetric biomolecular detection. ACS Nano, 2011, 5: 5383-5389

53 Su K H, Wei Q H, Zhang X, et al. Interparticle coupling effects on plasmon resonances of nanogold particles. Nano Lett, 2003, 3: 10871090

54 Tabor C, Murali R, Mahmoud M, et al. On the use of plasmonic nanoparticle pairs as a plasmon ruler: The dependence of the near-field dipole plasmon coupling on nanoparticle size and shape. J Phys Chem A, 2008, 113: 1946-1953

55 Yang L, Wang H, Yan B, et al. Calibration of silver plasmon rulers in the 1-25 $\mathrm{nm}$ separation range: Experimental indications of distinct plasmon coupling regimes. J Phys Chem C, 2010, 114: 4901-4908

56 Mock J J, Hill R T, Degiron A, et al. Distance-dependent plasmon resonant coupling between a gold nanoparticle and gold film. Nano Lett, 2008, 8: 2245-2252 
57 Rechberger W, Hohenau A, Leitner A, et al. Optical properties of two interacting gold nanoparticles. Opt Commun, 2003, 220: 137-141

58 Jain P K, Huang W, El-Sayed M A. On the universal scaling behavior of the distance decay of plasmon coupling in metal nanoparticle pairs: A plasmon ruler equation. Nano Lett, 2007, 7: 2080-2088

59 Zhang L, Chen H, Wang J, et al. Tetrakis(4-sulfonatophenyl)porphyrindirected assembly of gold nanocrystals: Tailoring the plasmon coupling through controllable gap distances. Small, 2010, 6: 2001-2009

60 Sönnichsen C, Reinhard B M, Liphardt J, et al. A molecular ruler based on plasmon coupling of single gold and silver nanoparticles. Nat Biotechnol, 2005, 23: 741-745

61 Reinhard B M, Sheikholeslami S, Mastroianni A, et al. Use of plasmon coupling to reveal the dynamics of DNA bending and cleavage by single ecorv restriction enzymes. Proc Natl Acad Sci USA, 2007, 104: 2667-2672

62 Jun Y W, Sheikholeslami S, Hostetter D R, et al. Continuous imaging of plasmon rulers in live cells reveals early-stage caspase-3 activation at the single-molecule level. Proc Natl Acad Sci USA, 2009, 106: $17735-17740$

63 Liu G L, Long Y T, Choi Y, et al. Quantized plasmon quenching dips nanospectroscopy via plasmon resonance energy transfer. Nat Methods, 2007, 4: 1015-1017

64 Choi Y, Park Y, Kang T, et al. Selective and sensitive detection of metal ions by plasmonic resonance energy transfer-based nanospectroscopy. Nat Nanotechnol, 2009, 4: 742-746

65 Qu W G, Deng B, Zhong S L, et al. Plasmonic resonance energy transfer-based nanospectroscopy for sensitive and selective detection of 2,4,6-trinitrotoluene (TNT). Chem Commun, 2011, 47: 1237-1239

66 Choi Y, Kang T, Lee L P. Plasmon resonance energy transfer (PRET)-based molecular imaging of cytochrome $c$ in living cells. Nano Lett, 2009, 9: 85-90

67 Novo C, Funston A M, Mulvaney P. Direct observation of chemical reactions on single gold nanocrystals using surface plasmon spectroscopy. Nat Nanotechnol, 2008, 3: 598-602

68 Zhang L, Li Y, Li D W, et al. Single gold nanoparticles as real-time optical probes for the detection of NADH-dependent intracellular metabolic enzymatic pathways. Angew Chem Int Ed, 2011, 123: 6921-6924

69 Qin L X, Jing C, Li Y, et al. Real-time monitoring of the aging of single plasmonic copper nanoparticles. Chem Commun, 2012, 48: 1511-1513

70 Chen L Q, Xiao S J, Hu P P, et al. Aptamer-mediated nanoparticle- based protein labeling platform for intracellular imaging and tracking endocytosis dynamics. Anal Chem, 2012, 84: 3099-3110

71 Joo K I, Lei Y, Lee C L, et al. Site-specific labeling of enveloped viruses with quantum dots for single virus tracking. ACS Nano, 2008, 2 : $1553-1562$

72 Jin R, Cao Y, Mirkin C A, et al. Photoinduced conversion of silver nanospheres to nanoprisms. Science, 2001, 294: 1901-1903

73 Huang T, Xu X H N. Synthesis and characterization of tunable rainbow colored colloidal silver nanoparticles using single-nanoparticle plasmonic microscopy and spectroscopy. J Mater Chem, 2010, 20: 9867-9876

74 El-Sayed I H, Huang X, El-Sayed M A. Surface plasmon resonance scattering and absorption of anti-EGFR antibody conjugated gold nanoparticles in cancer diagnostics: Applications in oral cancer. Nano Lett, 2005, 5: 829-834

75 Huang X, El-Sayed I H, Qian W, et al. Cancer cell imaging and photothermal therapy in the near-infrared region by using gold nanorods. J Am Chem Soc, 2006, 128: 2115-2120

76 Zhang L, Zhen S J, Sang Y, et al. Controllable preparation of metal nanoparticle/carbon nanotube hybrids as efficient dark field light scattering agents for cell imaging. Chem Commun, 2010, 46: 43034305

77 Huang T, Nallathamby P D, Gillet D, et al. Design and synthesis of single-nanoparticle optical biosensors for imaging and characterization of single receptor molecules on single living cells. Anal Chem, 2007, 79: 7708-7718

78 Kumar S, Harrison N, Richards-Kortum R, et al. Plasmonic nanosensors for imaging intracellular biomarkers in live cells. Nano Lett, 2007, 7: 1338-1343

79 Yang Y H, Nam J M. Single nanoparticle tracking-based detection of membrane receptor-ligand interactions. Anal Chem, 2009, 81: 2564-2568

80 Louit G, Asahi T, Tanaka G, et al. Spectral and 3-dimensional tracking of single gold nanoparticles in living cells studied by rayleigh light scattering microscopy. J Phys Chem C, 2009, 113: 1176611772

81 Chen L Q, Xiao S J, Peng L, et al. Aptamer-based silver nanoparticles used for intracellular protein imaging and single nanoparticle spectral analysis. J Phys Chem B, 2010, 114: 3655-3659

82 Rojas-Cherto M, Peironcely J E, Kasper P T, et al. Metabolite identification using automated comparison of high-resolution multistage mass spectral trees. Anal Chem, 2012, 84: 5524-5534

Open Access This article is distributed under the terms of the Creative Commons Attribution License which permits any use, distribution, and reproduction in any medium, provided the original author(s) and source are credited. 